\title{
Density Functional Theory Investigations of the Forbidden Double Insertion of Diazomethane into $\mathrm{Zr}-\mathrm{C}$ Bonds of $\mathrm{Cp}_{2} \mathrm{Zr}\left(\mathrm{CH}_{3}\right)_{2}$
}

\author{
P. Jeffrey Hay \\ Theoretical Division, Los Alamos National Laboratory \\ Los Alamos, NM 87545
}

Table of Contents

Calculated thermodynamic properties of stable species and transition states

Cartesian coordinates of computed structures 


\begin{tabular}{|c|c|c|c|c|c|c|c|c|}
\hline & $\mathrm{E}$ (elec) & kcal & $\mathrm{E}+\mathrm{ZPT}$ & kcal & Enthalpy & kcal & Free energy & kcal \\
\hline \multirow{2}{*}{\multicolumn{9}{|c|}{$\overline{\mathrm{R}}=\mathrm{H}$}} \\
\hline & & & & & & & & \\
\hline Cp2ZrMe2 & -513.40997 & & -513.16960 & & -513.15392 & & -513.21162 & \\
\hline $\mathrm{CH} 2 \mathrm{~N} 2$ & -148.68391 & & -148.65176 & & -148.64730 & & -148.67530 & \\
\hline $\mathrm{Cp} 2 \mathrm{ZrMe} 2+\mathrm{CH} 2 \mathrm{~N} 2$ & -662.09388 & 0.0 & -661.82136 & & -661.80122 & & -661.88692 & \\
\hline Adduct1 & -662.09965 & -3.6 & -661.82238 & -0.6 & -661.80401 & -1.8 & -661.86719 & 12.4 \\
\hline cnt TST1 & -662.09547 & -1.0 & -661.81819 & 2.0 & -661.79967 & 1.0 & -661.86435 & 14.2 \\
\hline $2-\mathrm{H}$ & -662.16631 & -45.4 & -661.88550 & -40.3 & -661.86681 & -41.2 & -661.93256 & -28.6 \\
\hline lat TST1 & -662.08521 & 5.4 & -661.80906 & 7.7 & -661.79002 & 7.0 & -661.85650 & 19.1 \\
\hline $1-\mathrm{H}$ & -662.16952 & -47.5 & -661.88842 & -42.1 & -661.86995 & -43.1 & -661.93426 & -29.7 \\
\hline $2-\mathrm{H}+\mathrm{CH} 2 \mathrm{~N} 2$ & -810.85022 & 0.0 & -810.53726 & 0.0 & -810.51410 & 0 & -810.60787 & 0.0 \\
\hline cnt TST1 & -810.82370 & 16.6 & -810.50751 & 18.7 & -810.48557 & 17.9 & -810.55719 & 31.8 \\
\hline $4-\mathrm{H}$ & -810.90657 & -35.4 & -810.58713 & -31.3 & -810.56481 & -31.8 & -810.63841 & -19.2 \\
\hline $1-\mathrm{H}+\mathrm{CH} 2 \mathrm{~N} 2$ & -810.85343 & 0.0 & -810.54018 & 0.0 & -810.51725 & 0.0 & -810.60956 & 0.0 \\
\hline lat TST2 & -810.82327 & 18.9 & -810.50814 & 20.1 & -810.48580 & 19.7 & -810.55827 & 32.2 \\
\hline $3-\mathrm{H}$ & -810.90858 & -34.6 & -810.58886 & -30.5 & -810.56665 & -31.0 & -810.63996 & -19.1 \\
\hline TST12 & -662.15217 & 8.9 & -661.87202 & 8.5 & -661.85402 & 8.0 & -661.91646 & 10.1 \\
\hline
\end{tabular}




\begin{tabular}{|c|c|c|c|c|c|c|c|c|}
\hline & $\mathrm{E}(\mathrm{Elec})$ & kcal & $E+Z P E$ & kcal & $\mathrm{H}(298)$ & kcal & $G(298)$ & kcal \\
\hline \multicolumn{9}{|l|}{$\mathrm{R}=\mathrm{Ph}$} \\
\hline Cp2ZrMe2 & -513.40997 & & -513.16960 & & -513.15392 & & -513.21162 & \\
\hline CPh2N2 & -610.69799 & & -610.49973 & & -610.48710 & & -610.53989 & \\
\hline Cp2ZrMe2 + CPh2N2 & -1124.10796 & 0.0 & -1123.66933 & 0.0 & -1123.64102 & 0.0 & -1123.75151 & 0.0 \\
\hline $2-\mathrm{Ph}$ & -1124.16892 & -38.3 & -1123.72366 & -34.1 & -1123.69686 & -35.0 & -1123.78016 & -18.0 \\
\hline $1-\mathrm{Ph}$ & -1124.17446 & -41.7 & -1123.72875 & -37.3 & -1123.70121 & -37.8 & -1123.78716 & -22.4 \\
\hline $2-\mathrm{Ph}+\mathrm{CH} 2 \mathrm{~N} 2$ & -1734.86691 & 0.0 & -1734.22339 & 0.0 & -1734.18396 & 0.0 & -1734.32005 & 0.0 \\
\hline cnt TST2 & -1734.83791 & 18.2 & -1734.19377 & 18.6 & -1734.15403 & 18.8 & -1734.26897 & 32.1 \\
\hline $4-\mathrm{Ph}$ & -1734.91953 & -33.0 & -1734.27062 & -29.6 & -1734.23026 & -29.1 & -1734.34609 & -16.3 \\
\hline $1-\mathrm{Ph}+\mathrm{CH} 2 \mathrm{~N} 2$ & -1734.87245 & 0.0 & -1734.22848 & 0.0 & -1734.18831 & 0.0 & -1734.32705 & 0.0 \\
\hline lat TST2 & -1734.83286 & 24.8 & -1734.18892 & 24.8 & -1734.14857 & 24.9 & -1734.26514 & 38.8 \\
\hline $3-\mathrm{Ph}$ & -1734.91551 & -27.0 & -1734.26675 & -24.0 & -1734.22733 & -24.5 & -1734.34029 & -8.3 \\
\hline
\end{tabular}




\begin{tabular}{crrc}
\multicolumn{4}{c}{ cp2zrme 2} \\
40 & 0.000022 & -0.280233 & 0.000836 \\
6 & 1.684436 & 1.585297 & 0.611046 \\
6 & 1.720164 & 1.459057 & -0.814205 \\
6 & 2.306434 & 0.198419 & -1.127702 \\
6 & 2.615793 & -0.458469 & 0.097670 \\
6 & 2.242171 & 0.400827 & 1.171344 \\
6 & -1.710951 & 1.494686 & -0.764603 \\
6 & -1.697439 & 1.553533 & 0.665466 \\
6 & -2.263933 & 0.343945 & 1.160149 \\
6 & -2.616103 & -0.465058 & 0.041457 \\
6 & -2.286986 & 0.248898 & -1.146792 \\
1 & 2.482507 & -0.192075 & -2.118441 \\
1 & 1.403277 & 2.206582 & -1.526706 \\
1 & 1.334221 & 2.444878 & 1.163177 \\
1 & 2.358677 & 0.188755 & 2.223548 \\
1 & 3.059502 & -1.438319 & 0.196683 \\
1 & -1.383173 & 2.275392 & -1.435284 \\
1 & -2.443736 & -0.095557 & -2.157778 \\
1 & -1.358687 & 2.386707 & 1.263437 \\
1 & -2.399442 & 0.083052 & 2.198894 \\
1 & -3.058828 & -1.449340 & 0.086378 \\
6 & -0.002301 & -1.808295 & -1.687157 \\
1 & 0.019248 & -1.355220 & -2.689020 \\
1 & 0.880202 & -2.457461 & -1.591380 \\
1 & -0.904708 & -2.432147 & -1.617598 \\
6 & 0.006412 & -1.721580 & 1.761702 \\
1 & 0.895636 & -2.365118 & 1.701242 \\
1 & 0.018050 & -1.221615 & 2.740866 \\
1 & -0.889803 & -2.356756 & 1.718296
\end{tabular}

$$
\begin{array}{cccc}
\multicolumn{4}{l}{\operatorname{ch} 2 \mathrm{n} 2} \\
7 & -1.311725 & 0.000005 & 0.000061 \\
7 & -0.148796 & -0.000011 & -0.000055 \\
6 & 1.147942 & -0.000002 & -0.000185 \\
1 & 1.668027 & -0.945573 & 0.000532 \\
1 & 1.667973 & 0.945631 & 0.000532
\end{array}
$$

cp2zrme(hyd) me-in 1-H

$\begin{array}{crrr}40 & 0.163026 & 0.001931 & 0.157678 \\ 7 & -1.977806 & -0.026947 & 0.455754 \\ 7 & -1.892540 & -0.027524 & -0.888970 \\ 6 & -3.261031 & -0.026127 & 1.131918 \\ 1 & -3.857458 & -0.918426 & 0.879611\end{array}$




$\begin{array}{rrrc}1 & -3.860192 & 0.862149 & 0.871265 \\ 1 & -3.075382 & -0.021009 & 2.206634 \\ 6 & -2.836277 & -0.035245 & -1.782942 \\ 6 & 1.541933 & 1.667653 & -1.319840 \\ 6 & 2.125577 & 1.719476 & -0.017312 \\ 6 & 1.177002 & 2.312508 & 0.866219 \\ 6 & 0.003838 & 2.613969 & 0.116327 \\ 6 & 0.232773 & 2.222686 & -1.236176 \\ 6 & 2.184633 & -1.604217 & -0.263730 \\ 6 & 1.359906 & -1.686041 & -1.427940 \\ 6 & 0.142942 & -2.331958 & -1.057126 \\ 6 & 0.201106 & -2.619117 & 0.334273 \\ 6 & 1.466522 & -2.174666 & 0.824705 \\ 1 & -3.890220 & -0.043059 & -1.516716 \\ 1 & -2.553630 & -0.034754 & -2.826840 \\ 1 & 1.323920 & 2.502652 & 1.917645 \\ 1 & 3.124237 & 1.403659 & 0.246061 \\ 1 & 2.017959 & 1.298941 & -2.216430 \\ 1 & -0.467157 & 2.323494 & -2.052248 \\ 1 & -0.898122 & 3.064910 & 0.502571 \\ 1 & 3.185326 & -1.200342 & -0.221833 \\ 1 & 1.818596 & -2.266242 & 1.840492 \\ 1 & 1.629231 & -1.366768 & -2.424040 \\ 1 & -0.688091 & -2.540537 & -1.714192 \\ 1 & -0.574265 & -3.091397 & 0.918998 \\ 6 & 0.151456 & -0.001824 & 2.461073 \\ 1 & 1.158447 & 0.045553 & 2.899789 \\ 1 & -0.424928 & 0.860489 & 2.821884 \\ 1 & -0.339182 & -0.917842 & 2.816049\end{array}$

\begin{tabular}{crrr}
\multicolumn{5}{c}{ cp2zr(me)(hyd) me-out $2-\mathrm{H}$} \\
40 & -0.160857 & 0.020960 & 0.153201 \\
7 & 1.804493 & -0.376345 & -0.678508 \\
7 & 2.101171 & -0.129593 & 0.610058 \\
6 & 2.831275 & -0.750721 & -1.631468 \\
1 & 3.339585 & -1.684958 & -1.338706 \\
1 & 3.603266 & 0.031049 & -1.729244 \\
1 & 2.362734 & -0.898924 & -2.606168 \\
6 & 3.245292 & -0.169214 & 1.225079 \\
6 & -1.858877 & 1.922380 & -0.359756 \\
6 & -1.042185 & 1.793198 & -1.530506 \\
6 & 0.264211 & 2.249697 & -1.202610 \\
6 & 0.271647 & 2.622227 & 0.169634 \\
6 & -1.046349 & 2.437463 & 0.685896 \\
6 & -1.773532 & -1.457084 & -1.276740
\end{tabular}




$\begin{array}{rrrc}6 & -2.395241 & -1.316323 & 0.005450 \\ 6 & -1.607286 & -2.024768 & 0.954730 \\ 6 & -0.485770 & -2.579450 & 0.271603 \\ 6 & -0.603183 & -2.244778 & -1.110910 \\ 1 & 4.173376 & -0.427530 & 0.720406 \\ 1 & 3.261394 & 0.063190 & 2.280677 \\ 1 & 1.112901 & 2.273468 & -1.869375 \\ 1 & -1.374917 & 1.467559 & -2.505138 \\ 1 & -2.915453 & 1.709332 & -0.296682 \\ 1 & -1.368349 & 2.651378 & 1.693076 \\ 1 & 1.122111 & 2.990662 & 0.724031 \\ 1 & -2.150343 & -1.069548 & -2.211777 \\ 1 & 0.087740 & -2.526975 & -1.891308 \\ 1 & -3.324146 & -0.805326 & 0.209902 \\ 1 & -1.815879 & -2.117050 & 2.008830 \\ 1 & 0.302074 & -3.168993 & 0.717799 \\ 6 & -0.238960 & 0.141350 & 2.454216 \\ 1 & 0.381173 & 0.982543 & 2.790737 \\ 1 & -1.253858 & 0.271984 & 2.855128 \\ 1 & 0.184967 & -0.782561 & 2.871201\end{array}$

\begin{tabular}{cccc}
\multicolumn{5}{c}{ cp2zr(hyd)2 Me-in $3-H$} \\
40 & -0.000102 & -0.131098 & 0.001442 \\
7 & -1.442763 & 1.476788 & -0.037163 \\
7 & -2.307785 & 0.454748 & 0.112952 \\
6 & -1.924168 & 2.843594 & -0.130213 \\
1 & -2.449678 & 3.156211 & 0.787433 \\
1 & -2.621163 & 2.970727 & -0.975277 \\
1 & -1.070964 & 3.503778 & -0.283998 \\
6 & -3.602294 & 0.493416 & 0.217828 \\
6 & 0.383242 & -2.160265 & 1.690346 \\
6 & -0.920363 & -1.637867 & 1.953918 \\
6 & -0.763587 & -0.347406 & 2.537111 \\
6 & 0.627729 & -0.062201 & 2.611634 \\
6 & 1.336516 & -1.190364 & 2.103206 \\
7 & 1.446787 & 1.467966 & -0.110928 \\
7 & 2.313463 & 0.436076 & -0.145181 \\
6 & 1.929389 & 2.837419 & -0.125040 \\
1 & 2.484214 & 3.066168 & -1.050051 \\
1 & 2.600443 & 3.039581 & 0.726511 \\
1 & 1.073841 & 3.509005 & -0.058359 \\
6 & 3.610151 & 0.466923 & -0.220818 \\
6 & -0.493594 & -2.283833 & -1.496778 \\
6 & 0.857901 & -1.891946 & -1.751480 \\
6 & 0.827512 & -0.660048 & -2.465392
\end{tabular}




$\begin{array}{lrrr}6 & -0.532263 & -0.274348 & -2.624647 \\ 6 & -1.348174 & -1.290373 & -2.044567 \\ 1 & -4.170278 & 1.420795 & 0.194420 \\ 1 & -4.131754 & -0.442736 & 0.335243 \\ 1 & 4.141903 & -0.475164 & -0.236202 \\ 1 & 4.178126 & 1.393227 & -0.271216 \\ 1 & -1.565532 & 0.307180 & 2.843513 \\ 1 & -1.858184 & -2.138248 & 1.763097 \\ 1 & 0.605742 & -3.132695 & 1.275919 \\ 1 & 2.408388 & -1.279463 & 2.021423 \\ 1 & 1.070658 & 0.845348 & 2.994944 \\ 1 & -0.810069 & -3.188977 & -0.999306 \\ 1 & -2.426082 & -1.288407 & -2.003790 \\ 1 & 1.744402 & -2.445516 & -1.478380 \\ 1 & 1.690284 & -0.102705 & -2.796937 \\ 1 & -0.886136 & 0.620564 & -3.115087\end{array}$

cp2zrhyd2 Me-out 4-H

$\begin{array}{cccc}40 & -0.202172 & -0.006294 & 0.002736 \\ 7 & 0.938451 & -1.560724 & 0.958670 \\ 7 & 1.724510 & -1.430221 & -0.136421 \\ 6 & 1.276192 & -2.519509 & 1.996632 \\ 1 & 2.269857 & -2.316992 & 2.428981 \\ 1 & 1.284135 & -3.552758 & 1.610735 \\ 1 & 0.532053 & -2.452019 & 2.790543 \\ 6 & 2.808862 & -2.081877 & -0.426746 \\ 6 & -2.413742 & -0.417523 & -1.474921 \\ 6 & -1.966580 & -1.701415 & -1.042174 \\ 6 & -0.739996 & -1.982718 & -1.712509 \\ 6 & -0.402947 & -0.859137 & -2.509968 \\ 6 & -1.441499 & 0.113606 & -2.360825 \\ 7 & 0.906695 & 1.563331 & -0.955864 \\ 7 & 1.693357 & 1.476036 & 0.143007 \\ 6 & 1.216380 & 2.506083 & -2.016652 \\ 1 & 2.220595 & 2.330184 & -2.436095 \\ 1 & 1.180812 & 3.549366 & -1.660832 \\ 1 & 0.481636 & 2.386436 & -2.813581 \\ 6 & 2.757049 & 2.165455 & 0.421348 \\ 6 & -2.370798 & 0.264515 & 1.556562 \\ 6 & -2.089510 & 1.559997 & 1.026562 \\ 6 & -0.876676 & 2.018581 & 1.616751 \\ 6 & -0.387467 & 0.998129 & 2.473471 \\ 6 & -1.316097 & -0.087588 & 2.438050 \\ 1 & 3.235487 & -2.844608 & 0.221412 \\ 1 & 3.313939 & -1.838762 & -1.351784\end{array}$




$\begin{array}{rrrr}1 & 3.268815 & 1.955226 & 1.350793 \\ 1 & 3.160570 & 2.929553 & -0.240154 \\ 1 & -0.144289 & -2.875337 & -1.597574 \\ 1 & -2.492790 & -2.369227 & -0.375391 \\ 1 & -3.329393 & 0.069532 & -1.177407 \\ 1 & -1.494005 & 1.071162 & -2.858250 \\ 1 & 0.487728 & -0.751278 & -3.110336 \\ 1 & -3.235386 & -0.341329 & 1.332668 \\ 1 & -1.242529 & -1.006478 & 3.000641 \\ 1 & -2.712328 & 2.119512 & 0.343850 \\ 1 & -0.390732 & 2.961424 & 1.416065 \\ 1 & 0.532593 & 1.029590 & 3.037030\end{array}$

$\begin{array}{crrr}\text { cnt } & \text { TST1 } & & \\ 40 & 0.168928 & 0.001647 & 0.095235 \\ 7 & -1.914463 & -0.091720 & 0.244231 \\ 7 & -3.113807 & -0.093943 & 0.478243 \\ 6 & -1.516625 & -0.084859 & -1.852199 \\ 1 & -2.110812 & 0.813982 & -2.019085 \\ 1 & -2.105404 & -0.991935 & -1.989299 \\ 1 & -0.645016 & -0.096915 & -2.513984 \\ 6 & -4.307419 & -0.111533 & -0.017330 \\ 6 & 2.310852 & -1.507339 & -0.063664 \\ 6 & 1.599069 & -1.683652 & -1.289808 \\ 6 & 0.391147 & -2.386172 & -0.995256 \\ 6 & 0.337633 & -2.607934 & 0.408307 \\ 6 & 1.527214 & -2.065385 & 0.985616 \\ 6 & 1.722969 & 1.732581 & -1.108826 \\ 6 & 2.094941 & 1.759721 & 0.270922 \\ 6 & 1.010214 & 2.316960 & 1.007972 \\ 6 & -0.037002 & 2.619100 & 0.093560 \\ 6 & 0.408480 & 2.270246 & -1.216332 \\ 1 & -4.475698 & -0.122063 & -1.090339 \\ 1 & -5.146431 & -0.112398 & 0.663015 \\ 1 & -0.353750 & -2.691765 & -1.714330 \\ 1 & 1.943995 & -1.395571 & -2.272582 \\ 1 & 3.281020 & -1.046228 & 0.045753 \\ 1 & 1.787909 & -2.088972 & 2.032289 \\ 1 & -0.461474 & -3.098860 & 0.943675 \\ 1 & 2.341570 & 1.398296 & -1.929020 \\ 1 & -0.147641 & 2.404288 & -2.131859 \\ 1 & 3.045945 & 1.453023 & 0.680506 \\ 1 & 0.987669 & 2.480082 & 2.073651 \\ 1 & -0.994710 & 3.049107 & 0.347266 \\ 6 & -0.174282 & -0.023144 & 2.394168\end{array}$




$\begin{array}{lrll}1 & -0.660397 & -0.963192 & 2.681320 \\ 1 & 0.777275 & 0.063492 & 2.935594 \\ 1 & -0.826452 & 0.807846 & 2.687912\end{array}$

$\begin{array}{crrr}\text { lat TST1 } & & & \\ 40 & 0.263102 & 0.039715 & 0.203935 \\ 7 & -1.761077 & -0.730327 & -0.149584 \\ 7 & -2.922245 & -0.821925 & -0.377176 \\ 6 & -1.181892 & -0.563057 & 2.158561 \\ 1 & -2.189183 & -0.982217 & 2.109143 \\ 1 & -1.205973 & 0.392261 & 2.691631 \\ 1 & -0.524020 & -1.271555 & 2.668480 \\ 6 & -4.203262 & -0.941824 & -0.324397 \\ 6 & 1.633865 & -1.134998 & -1.698470 \\ 6 & 0.591354 & -2.037154 & -1.351233 \\ 6 & 0.833909 & -2.501208 & -0.019707 \\ 6 & 2.034192 & -1.894732 & 0.442681 \\ 6 & 2.520630 & -1.033566 & -0.583385 \\ 6 & 0.986904 & 2.045774 & -1.289470 \\ 6 & 1.014604 & 2.555437 & 0.036148 \\ 6 & -0.330695 & 2.606525 & 0.516895 \\ 6 & -1.185223 & 2.138068 & -0.513123 \\ 6 & -0.373802 & 1.757948 & -1.626425 \\ 1 & -4.700675 & -1.011162 & 0.637081 \\ 1 & -4.768803 & -0.984254 & -1.244867 \\ 1 & 0.222788 & -3.203905 & 0.526644 \\ 1 & -0.233117 & -2.329827 & -1.983848 \\ 1 & 1.743081 & -0.628856 & -2.646330 \\ 1 & 3.417463 & -0.433142 & -0.534907 \\ 1 & 2.490548 & -2.047717 & 1.407836 \\ 1 & 1.841428 & 1.914275 & -1.936514 \\ 1 & -0.730583 & 1.389074 & -2.577292 \\ 1 & 1.892347 & 2.863705 & 0.582510 \\ 1 & -0.640604 & 2.947175 & 1.493575 \\ 1 & -2.261379 & 2.060841 & -0.456777 \\ 6 & 1.470768 & 0.486279 & 2.151449 \\ 1 & 1.525037 & -0.386853 & 2.811033 \\ 1 & 2.496106 & 0.775180 & 1.874318 \\ 1 & 1.016592 & 1.313185 & 2.711038\end{array}$

$\begin{array}{crrr}\text { cnt TST2 } & & & \\ 40 & 0.331972 & 0.099221 & 0.067939 \\ 7 & -0.993119 & -1.406217 & -0.840429 \\ 7 & -2.171878 & -1.450278 & -0.136048\end{array}$ 


$\begin{array}{rrrr}6 & -0.774569 & -2.477169 & -1.819007 \\ 1 & -1.542874 & -2.468059 & -2.609362 \\ 1 & -0.809639 & -3.472605 & -1.341785 \\ 1 & 0.199592 & -2.361915 & -2.287805 \\ 6 & -3.093236 & -2.350259 & -0.328823 \\ 6 & 1.866475 & -0.206850 & 2.183228 \\ 6 & 1.807203 & -1.496647 & 1.564948 \\ 6 & 0.478495 & -1.982909 & 1.692697 \\ 6 & -0.292156 & -1.000270 & 2.379335 \\ 6 & 0.571681 & 0.085069 & 2.701803 \\ 6 & 2.746500 & 0.336069 & -0.945154 \\ 6 & 2.151678 & 1.619106 & -1.088395 \\ 6 & 1.091938 & 1.512146 & -2.042935 \\ 6 & 1.027548 & 0.165954 & -2.476802 \\ 6 & 2.035342 & -0.574667 & -1.780657 \\ 1 & -3.051215 & -3.151183 & -1.065510 \\ 1 & -3.981966 & -2.280636 & 0.286709 \\ 1 & 0.103811 & -2.920236 & 1.311141 \\ 1 & 2.634418 & -2.021190 & 1.109252 \\ 1 & 2.747697 & 0.410279 & 2.285857 \\ 1 & 0.293695 & 0.972988 & 3.248361 \\ 1 & -1.346351 & -1.066290 & 2.597018 \\ 1 & 3.592648 & 0.095890 & -0.319662 \\ 1 & 2.270280 & -1.620361 & -1.913176 \\ 1 & 2.471760 & 2.525020 & -0.596560 \\ 1 & 0.446239 & 2.314153 & -2.367247 \\ 1 & 0.320178 & -0.231229 & -3.187453 \\ 6 & 0.043127 & 2.527821 & 0.957300 \\ 1 & -0.646531 & 2.687621 & 1.787071 \\ 1 & 1.068974 & 2.458557 & 1.331473 \\ 1 & -0.035284 & 3.312137 & 0.203030 \\ 7 & -1.404512 & 1.302192 & -0.048662 \\ 7 & -2.472659 & 1.860686 & -0.125542 \\ 6 & -3.251659 & 2.866118 & 0.088222 \\ 1 & -2.892042 & 3.755727 & 0.597461 \\ 1 & -4.277285 & 2.806722 & -0.246175\end{array}$

$\begin{array}{cccl}\text { lat TST2 } & & & \\ 40 & -0.027587 & 0.098587 & -0.034009 \\ 7 & 2.139528 & 0.413154 & 0.467595 \\ 7 & 3.267527 & 0.028508 & 0.560670 \\ 6 & 0.878582 & -0.090193 & 2.295270 \\ 1 & 1.845697 & 0.207061 & 2.703396 \\ 1 & 0.700690 & -1.154614 & 2.478248 \\ 1 & 0.101502 & 0.533766 & 2.742777\end{array}$




$\begin{array}{lrrr}6 & 4.326418 & -0.488543 & 1.086978 \\ 6 & -0.083646 & 2.157800 & -1.701058 \\ 6 & 0.805975 & 2.573372 & -0.673722 \\ 6 & 0.057781 & 2.697442 & 0.536942 \\ 6 & -1.308124 & 2.410476 & 0.240543 \\ 6 & -1.398410 & 2.066726 & -1.135362 \\ 7 & -1.677632 & -0.534278 & 1.227706 \\ 7 & -2.449446 & -0.423926 & 0.129090 \\ 6 & -2.245741 & -1.030900 & 2.471273 \\ 1 & -2.640395 & -2.054198 & 2.354637 \\ 1 & -3.072524 & -0.392276 & 2.823185 \\ 1 & -1.468464 & -1.042301 & 3.235045 \\ 6 & -3.713799 & -0.706273 & 0.017277 \\ 6 & 0.156579 & -1.109046 & -2.405700 \\ 6 & -0.782899 & -1.920380 & -1.707464 \\ 6 & -0.109718 & -2.545982 & -0.626077 \\ 6 & 1.259887 & -2.142423 & -0.664899 \\ 6 & 1.425214 & -1.256944 & -1.772555 \\ 1 & 4.299534 & -0.863264 & 2.105689 \\ 1 & 5.240325 & -0.518679 & 0.511695 \\ 1 & -4.182104 & -0.560115 & -0.947578 \\ 1 & -4.321187 & -1.070726 & 0.843061 \\ 1 & 0.454078 & 2.997241 & 1.496799 \\ 1 & 1.868549 & 2.733649 & -0.778087 \\ 1 & 0.174693 & 1.996307 & -2.737136 \\ 1 & -2.295676 & 1.778309 & -1.660084 \\ 1 & -2.127431 & 2.418992 & 0.943415 \\ 1 & -0.047727 & -0.531064 & -3.294969 \\ 1 & 2.346819 & -0.792231 & -2.088865 \\ 1 & -1.831330 & -2.019291 & -1.937589 \\ 1 & -0.552339 & -3.213522 & 0.098820 \\ 1 & 2.042342 & -2.471514 & 0.003802\end{array}$

$\begin{array}{crrr}\text { ph2cn2 } & & \\ 7 & 0.001623 & 3.290466 & 0.003034 \\ 7 & 0.000352 & 2.125965 & -0.000875 \\ 6 & -0.000007 & 0.811782 & 0.000092 \\ 6 & -1.317076 & 0.138862 & 0.027834 \\ 6 & -1.468979 & -1.123378 & 0.639639 \\ 6 & -2.718279 & -1.746867 & 0.681926 \\ 6 & -3.843917 & -1.124000 & 0.129573 \\ 6 & -3.706748 & 0.133549 & -0.470376 \\ 6 & -2.458620 & 0.756488 & -0.526444 \\ 1 & -0.610754 & -1.605969 & 1.092859 \\ 1 & -2.813200 & -2.717157 & 1.159506\end{array}$




$\begin{array}{rrrr}1 & -4.813272 & -1.609999 & 0.167505 \\ 1 & -4.570786 & 0.627045 & -0.904304 \\ 1 & -2.364718 & 1.724011 & -1.010380 \\ 6 & 1.317056 & 0.138307 & -0.027319 \\ 6 & 2.459362 & 0.757030 & 0.523325 \\ 6 & 3.707345 & 0.133596 & 0.466623 \\ 6 & 3.843329 & -1.125247 & -0.130479 \\ 6 & 2.716774 & -1.749129 & -0.679606 \\ 6 & 1.467762 & -1.125300 & -0.636783 \\ 1 & 2.366581 & 1.725730 & 1.005004 \\ 1 & 4.572150 & 0.627871 & 0.897991 \\ 1 & 4.812490 & -1.611601 & -0.169041 \\ 1 & 2.811069 & -2.720577 & -1.154891 \\ 1 & 0.608596 & -1.608525 & -1.087398\end{array}$

\begin{tabular}{crrr}
\multicolumn{5}{c}{ c 2zrme(hydph) } & $1-\mathrm{Ph}$ \\
40 & 1.879782 & -0.428187 & 0.003111 \\
7 & 0.077944 & -1.584468 & -0.078576 \\
7 & -0.456267 & -0.345480 & -0.038748 \\
6 & -0.636902 & -2.851378 & -0.165180 \\
1 & -1.288768 & -2.902546 & -1.046301 \\
1 & -1.250544 & -3.051274 & 0.722078 \\
1 & 0.126148 & -3.628102 & -0.245437 \\
6 & -1.692222 & 0.108689 & -0.031266 \\
6 & 2.525184 & 1.442435 & 1.721090 \\
6 & 3.587117 & 0.488751 & 1.766180 \\
6 & 3.071039 & -0.716748 & 2.323844 \\
6 & 1.690560 & -0.514064 & 2.613766 \\
6 & 1.355540 & 0.823018 & 2.249892 \\
6 & 3.616788 & 0.751069 & -1.572105 \\
6 & 2.415593 & 1.518097 & -1.664280 \\
6 & 1.434988 & 0.719484 & -2.326235 \\
6 & 2.018829 & -0.543779 & -2.617555 \\
6 & 3.370085 & -0.522782 & -2.156818 \\
1 & 3.629597 & -1.622619 & 2.499184 \\
1 & 4.610438 & 0.659914 & 1.465735 \\
1 & 2.602597 & 2.465151 & 1.382390 \\
1 & 0.385121 & 1.286037 & 2.349422 \\
1 & 1.017948 & -1.244051 & 3.039180 \\
1 & 4.554027 & 1.087395 & -1.154159 \\
1 & 4.081293 & -1.329154 & -2.245624 \\
1 & 2.289657 & 2.542599 & -1.346118 \\
1 & 0.422161 & 1.020281 & -2.549645 \\
1 & 1.528605 & -1.373885 & -3.104085 \\
6 & 2.812340 & -2.539208 & -0.016415
\end{tabular}




$\begin{array}{lrrr}1 & 3.910018 & -2.525529 & 0.044798 \\ 1 & 2.425726 & -3.117059 & 0.833574 \\ 1 & 2.524516 & -3.051701 & -0.944259 \\ 6 & -2.892570 & -0.797778 & -0.028201 \\ 6 & -3.591856 & -1.063585 & -1.219949 \\ 6 & -4.719950 & -1.890943 & -1.215890 \\ 6 & -5.172857 & -2.456086 & -0.017287 \\ 6 & -4.495450 & -2.185139 & 1.177178 \\ 6 & -3.365826 & -1.358584 & 1.172085 \\ 1 & -3.245840 & -0.620728 & -2.148967 \\ 1 & -5.243296 & -2.093828 & -2.145055 \\ 1 & -6.048078 & -3.097982 & -0.013892 \\ 1 & -4.845683 & -2.613992 & 2.110907 \\ 1 & -2.845912 & -1.142779 & 2.100679 \\ 6 & -1.928046 & 1.570894 & -0.004701 \\ 6 & -0.875151 & 2.509228 & -0.108598 \\ 6 & -1.123874 & 3.881463 & -0.077040 \\ 6 & -2.431498 & 4.366308 & 0.057011 \\ 6 & -3.485726 & 3.453439 & 0.160729 \\ 6 & -3.240566 & 2.078032 & 0.131207 \\ 1 & 0.139823 & 2.154099 & -0.218405 \\ 1 & -0.293485 & 4.576493 & -0.161275 \\ 1 & -2.622965 & 5.434221 & 0.078931 \\ 1 & -4.505846 & 3.809831 & 0.267040 \\ 1 & -4.073689 & 1.391326 & 0.217368\end{array}$

\begin{tabular}{|c|c|c|c|}
\hline & dph) & 2-Ph & \\
\hline 40 & 1.870335 & -0.017065 & -0.076939 \\
\hline 7 & 0.211609 & -1.215632 & 0.558589 \\
\hline 7 & -0.504712 & -0.128037 & 0.189059 \\
\hline 6 & -0.285682 & -2.348175 & 1.326601 \\
\hline 1 & -0.844915 & -3.075135 & 0.723126 \\
\hline 1 & -0.945387 & -2.019047 & 2.142174 \\
\hline 1 & 0.584426 & -2.850554 & 512 \\
\hline 6 & -1.807592 & 0.066136 & 0.105002 \\
\hline 6 & 3.802019 & 1.141338 & 1.206606 \\
\hline 6 & 3.459060 & -0.023374 & 558 \\
\hline 6 & 2.173340 & 0.189829 & 2.537847 \\
\hline 6 & 1.711901 & 1.469421 & 2.116389 \\
\hline 6 & 2.725090 & 2.063414 & 1.306325 \\
\hline 6 & 3.997566 & -1.283327 & -0.964981 \\
\hline 6 & 3.586754 & -0.433377 & -2.026849 \\
\hline 6 & 2.336070 & -0.921239 & -2.521620 \\
\hline 6 & 1.976826 & -2.061407 & -1.758563 \\
\hline 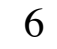 & 2.987328 & -2.273634 & -0.771848 \\
\hline
\end{tabular}




$\begin{array}{lrrr}1 & 1.636009 & -0.501809 & 3.169650 \\ 1 & 4.083482 & -0.891545 & 2.119895 \\ 1 & 4.727984 & 1.303176 & 0.674480 \\ 1 & 2.680401 & 3.037676 & 0.845417 \\ 1 & 0.769676 & 1.919599 & 2.389103 \\ 1 & 4.914837 & -1.196740 & -0.401984 \\ 1 & 3.013392 & -3.077263 & -0.049900 \\ 1 & 4.131097 & 0.415992 & -2.411805 \\ 1 & 1.771542 & -0.496193 & -3.337262 \\ 1 & 1.079835 & -2.649711 & -1.879505 \\ 6 & 1.414589 & 1.792730 & -1.420092 \\ 1 & 0.875109 & 2.576765 & -0.879391 \\ 1 & 2.338494 & 2.223464 & -1.832257 \\ 1 & 0.779896 & 1.473289 & -2.257966 \\ 6 & -2.295577 & 1.437290 & -0.185083 \\ 6 & -3.469976 & 1.636129 & -0.943225 \\ 6 & -3.934716 & 2.923574 & -1.222551 \\ 6 & -3.243538 & 4.044266 & -0.746754 \\ 6 & -2.082330 & 3.862597 & 0.014024 \\ 6 & -1.617799 & 2.575819 & 0.295390 \\ 1 & -4.011455 & 0.777196 & -1.323518 \\ 1 & -4.834852 & 3.051175 & -1.816146 \\ 1 & -3.607212 & 5.044183 & -0.961266 \\ 1 & -1.543206 & 4.723907 & 0.396703 \\ 1 & -0.727272 & 2.438219 & 0.894201 \\ 6 & -2.820793 & -1.027559 & 0.199851 \\ 6 & -2.743432 & -2.153972 & -0.643860 \\ 6 & -3.722794 & -3.152914 & -0.597466 \\ 6 & -4.797052 & -3.044146 & 0.292112 \\ 6 & -4.892584 & -1.923231 & 1.128187 \\ 6 & -3.919908 & -0.922177 & 1.076778 \\ 1 & -1.921129 & -2.232289 & -1.348476 \\ 1 & -3.648800 & -4.010127 & -1.259585 \\ 1 & -5.555757 & -3.819330 & 0.329816 \\ 1 & -5.725381 & -1.830072 & 1.818344 \\ 1 & -4.003121 & -0.050593 & 1.718013\end{array}$

\begin{tabular}{cccc}
\multicolumn{5}{c}{ cp2zr(hydph)2 me-in 3-Ph } \\
40 & -0.002370 & -0.546195 & 0.003606 \\
7 & 1.363573 & 1.083883 & -0.264199 \\
7 & 2.354928 & 0.175861 & -0.120965 \\
6 & 1.510559 & 2.418164 & -0.841726 \\
1 & 2.169655 & 2.398697 & -1.720469 \\
1 & 1.912695 & 3.159367 & -0.140249 \\
1 & 0.514281 & 2.734414 & -1.156926
\end{tabular}




\begin{tabular}{lrrr}
6 & 3.666100 & 0.312549 & -0.114423 \\
6 & -0.941045 & -2.346920 & -1.675651 \\
6 & 0.473178 & -2.553441 & -1.722928 \\
6 & 1.059894 & -1.418181 & -2.339528 \\
6 & 0.014090 & -0.505455 & -2.676492 \\
6 & -1.220534 & -1.094574 & -2.296376 \\
7 & -1.363214 & 1.087773 & 0.274341 \\
7 & -2.352938 & 0.178588 & 0.125932 \\
6 & -1.513163 & 2.418359 & 0.859276 \\
1 & -2.175273 & 2.392827 & 1.735646 \\
1 & -1.913693 & 3.163530 & 0.161092 \\
1 & -0.518233 & 2.733660 & 1.179725 \\
6 & -3.664253 & 0.313507 & 0.114224 \\
6 & 0.934236 & -2.349851 & 1.680946 \\
6 & -0.480694 & -2.550491 & 1.731679 \\
6 & -1.061075 & -1.412707 & 2.349775 \\
6 & -0.010574 & -0.504436 & 2.684120 \\
6 & 1.220539 & -1.098680 & 2.300861 \\
1 & 2.113034 & -1.260116 & -2.513243 \\
1 & 0.994923 & -3.430135 & -1.366908 \\
1 & -1.677691 & -3.043470 & -1.305552 \\
1 & -2.200605 & -0.660996 & -2.418705 \\
1 & 0.141407 & 0.449959 & -3.164468 \\
1 & 1.666973 & -3.049661 & 1.309302 \\
1 & 2.202663 & -0.668971 & 2.420233 \\
1 & -1.006853 & -3.425135 & 1.377126 \\
1 & -2.113082 & -1.250198 & 2.526460 \\
1 & -0.132615 & 0.451688 & 3.172040 \\
6 & 4.366494 & 1.637741 & -0.137268 \\
6 & 5.269466 & 1.958779 & -1.169946 \\
6 & 4.184345 & 2.563277 & 0.907640 \\
6 & 5.949023 & 3.179991 & -1.171150 \\
1 & 5.433002 & 1.245210 & -1.971502 \\
6 & 4.869441 & 3.784107 & 0.909717 \\
1 & 3.509041 & 2.318417 & 1.721935 \\
6 & 5.749984 & 4.097989 & -0.131380 \\
1 & 6.632838 & 3.415650 & -1.980708 \\
1 & 4.718487 & 4.485023 & 1.724930 \\
1 & 6.280306 & 5.044991 & -0.130854 \\
6 & 4.501117 & -0.907186 & 0.029506 \\
6 & 4.005858 & -2.190506 & -0.289114 \\
6 & 5.827565 & -0.816519 & 0.507503 \\
6 & 4.796821 & -3.329872 & -0.130510 \\
1 & 2.997992 & -2.281962 & -0.666977 \\
& 6.617597 & -1.958614 & 0.664168 \\
\hline & & & \\
6
\end{tabular}




$\begin{array}{lrrr}6 & 6.109264 & -3.222705 & 0.346633 \\ 1 & 4.391690 & -4.303595 & -0.389996 \\ 1 & 7.631564 & -1.858694 & 1.039451 \\ 1 & 6.725003 & -4.108551 & 0.465027 \\ 6 & -4.497276 & -0.906956 & -0.034980 \\ 6 & -5.816991 & -0.818492 & -0.531626 \\ 6 & -4.006438 & -2.188545 & 0.296691 \\ 6 & -6.604757 & -1.961351 & -0.693770 \\ 1 & -6.223592 & 0.148607 & -0.802340 \\ 6 & -4.795210 & -3.328764 & 0.132839 \\ 1 & -3.003915 & -2.278064 & 0.688931 \\ 6 & -6.100849 & -3.223871 & -0.363004 \\ 1 & -7.613405 & -1.863363 & -1.083599 \\ 1 & -4.393856 & -4.301173 & 0.402870 \\ 1 & -6.714976 & -4.110267 & -0.485546 \\ 6 & -4.366218 & 1.637684 & 0.134931 \\ 6 & -4.173378 & 2.568313 & -0.903584 \\ 6 & -5.281833 & 1.952509 & 1.158388 \\ 6 & -4.860708 & 3.787874 & -0.908244 \\ 1 & -3.488081 & 2.328372 & -1.710957 \\ 6 & -5.963453 & 3.172529 & 1.157139 \\ 1 & -5.453597 & 1.234976 & 1.954649 \\ 6 & -5.753970 & 4.095513 & 0.123853 \\ 1 & -4.701553 & 4.492675 & -1.718528 \\ 1 & -6.657069 & 3.403375 & 1.959710 \\ 1 & -6.285971 & 5.041567 & 0.121340\end{array}$

\begin{tabular}{crcc}
\multicolumn{5}{c}{ cp2zr(ph2hyd)2 } & $4-\mathrm{Ph}$ \\
40 & 0.011016 & 2.209028 & -0.008291 \\
7 & -1.714006 & 1.138437 & 0.682730 \\
7 & -1.455316 & 0.276575 & -0.327642 \\
6 & -2.897923 & 1.128349 & 1.540290 \\
1 & -2.926264 & 0.265017 & 2.216865 \\
1 & -3.827078 & 1.128534 & 0.957332 \\
1 & -2.861821 & 2.042867 & 2.136620 \\
6 & -2.043053 & -0.830425 & -0.724395 \\
6 & -1.485329 & -1.541456 & -1.902879 \\
6 & -2.104128 & -2.711366 & -2.397355 \\
1 & -2.990236 & -3.096949 & -1.909070 \\
6 & -1.589924 & -3.387391 & -3.507892 \\
1 & -2.088073 & -4.284203 & -3.863723 \\
6 & -0.442500 & -2.920515 & -4.155416 \\
1 & -0.046864 & -3.443853 & -5.020188 \\
6 & 0.191612 & -1.768588 & -3.669539 \\
1 & 1.087481 & -1.396830 & -4.158840
\end{tabular}




\begin{tabular}{|c|c|c|c|}
\hline 6 & -0.315828 & -1.090223 & -2.560202 \\
\hline 1 & 0.193012 & -0.212471 & -2.184285 \\
\hline 6 & -3.243457 & -1.431332 & -0.043256 \\
\hline 6 & -3.087958 & -2.257728 & 1.083903 \\
\hline 1 & -2.092338 & -2.446644 & 1.472473 \\
\hline 6 & -4.203999 & -2.831004 & 1.705459 \\
\hline 1 & -4.067751 & -3.463627 & 2.57719 \\
\hline 6 & -5.489049 & -2.593348 & 1.20508 \\
\hline 1 & -6.353658 & -3.037526 & \\
\hline 6 & -5.654210 & -1.783919 & 0.07 \\
\hline 1 & -6.647783 & -1.598818 & -0.3 \\
\hline 6 & -4.539116 & -1.212321 & -0.548470 \\
\hline 1 & -4.668094 & -0.590249 & -1. \\
\hline 6 & -0.831424 & 4.371575 & -1.282633 \\
\hline 6 & -1.852048 & 3.4 & -1.5 \\
\hline 6 & -1.322541 & 2.396439 & -2.362881 \\
\hline 6 & 0.025964 & 2.726228 & -2.65 \\
\hline 6 & 0.345931 & 3.934969 & -1.960485 \\
\hline 7 & 1.722412 & 234 & -0.6 \\
\hline 7 & 1.454588 & 872 & 0.322882 \\
\hline 6 & 2.904183 & 1.085580 & -1.5 \\
\hline 1 & 2.926468 & 0.20 & -2.213716 \\
\hline 1 & 3.835454 & 1.093843 & -0.974532 \\
\hline 1 & 2.869028 & 1.988515 & -2.167772 \\
\hline 6 & 2.031979 & -0.840029 & 0.732381 \\
\hline 6 & 1.470992 & -1.538595 & 1.915841 \\
\hline 6 & 2.074105 & -2.718158 & 2.407822 \\
\hline 1 & 2.946439 & -3.122812 & 1.909909 \\
\hline 6 & 1.563681 & -3.3 & 3.529708 \\
\hline 1 & 2.049387 & -4.282749 & 3.882946 \\
\hline 6 & 0.437960 & -2.882891 & 4.194287 \\
\hline 1 & 0.045921 & -3.393019 & 5.068545 \\
\hline 6 & -0.177792 & -1.71 & 3.713855 \\
\hline 1 & -1.054906 & -1.323248 & 4.217967 \\
\hline 6 & 0.325152 & -1.057780 & 2.591901 \\
\hline 1 & -0.166951 & -0.167044 & 2.224699 \\
\hline 6 & 3.221116 & -1.459982 & 0.048734 \\
\hline 6 & 3.050161 & -2.308856 & -1.059087 \\
\hline 1 & 2.049425 & -2.501231 & -1.433380 \\
\hline 6 & 4.157653 & -2.897696 & -1.681371 \\
\hline 1 & 4.010056 & -3.547507 & -2.538448 \\
\hline 6 & 5.449004 & -2.652554 & -1.200919 \\
\hline 1 & 6.306872 & -3.108699 & -1.684975 \\
\hline 6 & 5.629362 & -1.820207 & -0.088823 \\
\hline 1 & 6.627884 & -1.629459 & 0.29227 \\
\hline 6 & 4.522924 & -1.232664 & 0.5340 \\
\hline
\end{tabular}




$\begin{array}{rrrr}1 & 4.662972 & -0.593885 & 1.401051 \\ 6 & 0.885120 & 4.373027 & 1.238992 \\ 6 & 1.890242 & 3.402529 & 1.496247 \\ 6 & 1.338264 & 2.417073 & 2.366723 \\ 6 & -0.011040 & 2.767928 & 2.628327 \\ 6 & -0.303789 & 3.973407 & 1.921227 \\ 1 & -1.845900 & 1.521103 & -2.712707 \\ 1 & -2.860266 & 3.449605 & -1.123251 \\ 1 & -0.926190 & 5.277139 & -0.702526 \\ 1 & 1.288220 & 4.462882 & -2.000132 \\ 1 & 0.691832 & 2.158820 & -3.283741 \\ 1 & 0.999881 & 5.266733 & 0.646310 \\ 1 & -1.235706 & 4.520096 & 1.950982 \\ 1 & -0.694031 & 2.220737 & 3.259976 \\ 1 & 1.845644 & 1.539688 & 2.735239 \\ 1 & 2.901757 & 3.417958 & 1.117555\end{array}$

\begin{tabular}{cccc}
\multicolumn{4}{l}{ cnt TST2 Ph } \\
40 & -0.074225 & -2.412178 & -0.097990 \\
7 & -1.698027 & -1.068933 & -0.757265 \\
7 & -1.649209 & -0.115635 & 0.202197 \\
6 & -2.741243 & -1.070911 & -1.797361 \\
1 & -2.809594 & -0.099467 & -2.300437 \\
1 & -3.735284 & -1.307834 & -1.393843 \\
1 & -2.487162 & -1.823928 & -2.540683 \\
6 & -2.423794 & 0.916949 & 0.461143 \\
6 & -0.149877 & -4.226752 & 1.831277 \\
6 & -1.502792 & -4.098126 & 1.389063 \\
6 & -1.958800 & -2.801236 & 1.746638 \\
6 & -0.897915 & -2.121094 & 2.411072 \\
6 & 0.213352 & -3.008251 & 2.476934 \\
6 & -0.072037 & -4.734194 & -1.360783 \\
6 & 1.259075 & -4.242114 & -1.449084 \\
6 & 1.248603 & -3.092924 & -2.301187 \\
6 & -0.087216 & -2.852982 & -2.698476 \\
6 & -0.916227 & -3.857417 & -2.097822 \\
1 & -2.933483 & -2.389501 & 1.534755 \\
1 & -2.086752 & -4.859562 & 0.892987 \\
1 & 0.471153 & -5.106232 & 1.737587 \\
1 & 1.162179 & -2.800156 & 2.947798 \\
1 & -0.935575 & -1.113220 & 2.793358 \\
1 & -0.386801 & -5.612709 & -0.819763 \\
1 & -1.981102 & -3.974206 & -2.232706 \\
1 & 2.133495 & -4.692167 & -1.003043 \\
1 & 2.105775 & -2.493518 & -2.566613
\end{tabular}




$\begin{array}{lrrr}1 & -0.419862 & -2.042022 & -3.327529 \\ 6 & 2.441127 & -2.110781 & 0.553182 \\ 1 & 2.638090 & -1.482702 & 1.420225 \\ 1 & 2.401189 & -3.165666 & 0.839721 \\ 1 & 3.177518 & -1.958200 & -0.234076 \\ 7 & 1.125331 & -0.691075 & -0.362876 \\ 7 & 1.621331 & 0.397857 & -0.558316 \\ 6 & 2.564815 & 1.296353 & -0.562825 \\ 6 & 2.281983 & 2.611367 & -1.171383 \\ 6 & 3.268788 & 3.617947 & -1.195850 \\ 6 & 3.005388 & 4.860878 & -1.779502 \\ 6 & 1.756574 & 5.126523 & -2.349784 \\ 6 & 0.766955 & 4.133049 & -2.327901 \\ 6 & 1.022362 & 2.892368 & -1.746347 \\ 1 & 4.241335 & 3.427126 & -0.756967 \\ 1 & 3.779549 & 5.622090 & -1.786177 \\ 1 & 1.553928 & 6.092069 & -2.801938 \\ 1 & -0.207571 & 4.329136 & -2.765020 \\ 1 & 0.250199 & 2.130767 & -1.722890 \\ 6 & 3.909867 & 0.985649 & 0.030886 \\ 6 & 4.120921 & 1.092603 & 1.417188 \\ 6 & 5.371282 & 0.794037 & 1.969943 \\ 6 & 6.427571 & 0.392726 & 1.142663 \\ 6 & 6.230780 & 0.296261 & -0.240401 \\ 6 & 4.980174 & 0.592788 & -0.793412 \\ 1 & 3.303642 & 1.413070 & 2.055954 \\ 1 & 5.521609 & 0.877025 & 3.041764 \\ 1 & 7.397454 & 0.161006 & 1.571401 \\ 1 & 7.047787 & -0.009831 & -0.886228 \\ 1 & 4.827091 & 0.522226 & -1.865956 \\ 6 & -2.003459 & 1.775340 & 1.602296 \\ 6 & -2.886314 & 2.717956 & 2.171639 \\ 6 & -2.493919 & 3.513834 & 3.252717 \\ 6 & -1.210501 & 3.393304 & 3.794217 \\ 6 & -0.319523 & 2.466034 & 3.235344 \\ 6 & -0.706254 & 1.672305 & 2.155498 \\ 1 & -3.887771 & 2.824819 & 1.771502 \\ 1 & -3.196650 & 4.227758 & 3.672434 \\ 1 & -0.905710 & 4.013570 & 4.631308 \\ 1 & 0.685274 & 2.370196 & 3.638244 \\ 1 & -0.013405 & 0.968120 & 1.713011 \\ 6 & -3.690770 & 1.311392 & -0.249863 \\ 6 & -3.705349 & 2.424221 & -1.113254 \\ 6 & -6.882597 & 2.816810 & -1.757879 \\ & -6.079146 & 1.019575 & -0.664111\end{array}$




$\begin{array}{rrrr}6 & -4.898629 & 0.626541 & -0.021776 \\ 1 & -2.785863 & 2.979238 & -1.273726 \\ 1 & -4.872465 & 3.671134 & -2.428008 \\ 1 & -6.987804 & 2.416832 & -2.037622 \\ 1 & -7.000869 & 0.476516 & -0.478311 \\ 1 & -4.910147 & -0.213190 & 0.666794\end{array}$

lat TST2

$\begin{array}{rrrr}40 & 0.144237 & -0.473294 & 0.384981 \\ 7 & -2.088565 & -0.383436 & 0.625928 \\ 7 & -3.233100 & -0.287149 & 0.308822 \\ 6 & -1.001300 & 1.590709 & 1.128800 \\ 1 & -2.033282 & 1.745979 & 1.436947 \\ 1 & -0.768753 & 2.241327 & 0.280668 \\ 1 & -0.345281 & 1.778616 & 1.981331 \\ 6 & -4.440951 & 0.134315 & 0.035116 \\ 6 & 0.122574 & -2.903235 & 1.482888 \\ 6 & -0.820607 & -2.198946 & 2.272715 \\ 6 & -0.136554 & -1.149196 & 2.953644 \\ 6 & 1.249480 & -1.245207 & 2.623430 \\ 6 & 1.409073 & -2.318378 & 1.704165 \\ 7 & 1.698841 & 1.075119 & 0.515953 \\ 7 & 2.657945 & 0.168450 & 0.232789 \\ 6 & 1.999242 & 2.477476 & 0.809647 \\ 1 & 2.195757 & 3.077296 & -0.089478 \\ 1 & 2.881541 & 2.556795 & 1.458666 \\ 1 & 1.144218 & 2.911819 & 1.327174 \\ 6 & 3.951897 & 0.305857 & 0.009929 \\ 6 & 0.388829 & -2.041859 & -1.748338 \\ 6 & 1.241960 & -0.944379 & -2.055082 \\ 6 & 0.428832 & 0.211811 & -2.218613 \\ 6 & -0.928601 & -0.170550 & -2.038110 \\ 6 & -0.957722 & -1.567834 & -1.750221 \\ 1 & -0.586746 & -0.436605 & 3.628130 \\ 1 & -1.881460 & -2.392695 & 2.307026 \\ 1 & -0.084108 & -3.758874 & 0.856427 \\ 1 & 2.331073 & -2.646054 & 1.254727 \\ 1 & 2.032747 & -0.599283 & 2.988959 \\ 1 & 0.701809 & -3.064236 & -1.594024 \\ 1 & -1.841051 & -2.167315 & -1.589971 \\ 1 & 2.317115 & -0.984947 & -2.115630 \\ 1 & 0.782553 & 1.207787 & -2.441618 \\ 1 & -1.786678 & 0.480371 & -2.121964 \\ 6 & -4.762157 & 1.581967 & 0.238791 \\ 6 & -4.723809 & 2.150479 & 1.526855\end{array}$




$\begin{array}{lrrr}6 & -5.104946 & 2.400865 & -0.858044 \\ 6 & -5.013941 & 3.506063 & 1.714400 \\ 1 & -4.475731 & 1.524264 & 2.380676 \\ 6 & -5.401661 & 3.753878 & -0.667736 \\ 1 & -5.134773 & 1.971990 & -1.855883 \\ 6 & -5.354432 & 4.311002 & 0.618379 \\ 1 & -4.979337 & 3.930081 & 2.714074 \\ 1 & -5.662115 & 4.374036 & -1.522436 \\ 1 & -5.581435 & 5.362354 & 0.764164 \\ 6 & -5.439383 & -0.841574 & -0.443713 \\ 6 & -6.791067 & -0.462180 & -0.633932 \\ 6 & -5.080503 & -2.189028 & -0.709253 \\ 6 & -7.735723 & -1.388370 & -1.085728 \\ 1 & -7.102063 & 0.557351 & -0.421083 \\ 6 & -6.028744 & -3.108570 & -1.154367 \\ 1 & -4.049427 & -2.511208 & -0.558948 \\ 6 & -7.365360 & -2.715347 & -1.351028 \\ 1 & -8.768711 & -1.070019 & -1.224164 \\ 1 & -5.725343 & -4.136441 & -1.352355 \\ 1 & -8.101014 & -3.431599 & -1.701739 \\ 6 & 4.766431 & -0.942930 & -0.089592 \\ 6 & 4.779701 & -1.894178 & 0.948283 \\ 6 & 5.580364 & -1.177409 & -1.217663 \\ 6 & 5.548348 & -3.059518 & 0.847791 \\ 1 & 4.202947 & -1.701354 & 1.845681 \\ 6 & 6.344982 & -2.342361 & -1.321307 \\ 1 & 5.605625 & -0.441356 & -2.015200 \\ 6 & 6.328566 & -3.291795 & -0.290373 \\ 1 & 5.546944 & -3.777160 & 1.662918 \\ 1 & 6.953361 & -2.509125 & -2.205271 \\ 1 & 6.926044 & -4.194638 & -0.368764 \\ 6 & 4.681884 & 1.575419 & -0.297012 \\ 6 & 5.886437 & 1.881527 & 0.372667 \\ 6 & 4.251057 & 2.443065 & -1.324192 \\ 6 & 6.612958 & 3.030565 & 0.053976 \\ 1 & 6.245398 & 1.210268 & 1.148218 \\ 6 & 4.982753 & 3.592058 & -1.647623 \\ 1 & 3.349579 & 2.202643 & -1.881984 \\ 6 & 6.162310 & 3.893601 & -0.956285 \\ 1 & 7.530890 & 3.253434 & 0.591215 \\ 1 & 4.634325 & 4.245214 & -2.443811 \\ 1 & 6.728733 & 4.785264 & -1.205942\end{array}$

